\title{
Ulcerated gout masquerading as a non healing diabetic foot ulcer: a case series
}

\author{
WINSTON CRASTO, RAJESH JOGIA, STEPHEN JACKSON, KAUSTUBH NISAL, KATH HIGGINS, \\ MARIE-FRANCE KONG
}

\begin{abstract}
Gout has made a significant resurgence in recent years affecting people with type 2 diabetes, hypertension and chronic kidney disease. Although uncommon, ulcerated gout should be considered in patients presenting with a non healing diabetic foot ulcer, particularly if the first hallux is involved with bony involvement seen on imaging studies. A prior history of gout or hyperuricaemia can be helpful. Early recognition of ulcerated gout masquerading as diabetic foot infection/osteomyelitis and prompt institution of aggressive medical treatment can preserve joint integrity and aid prompt healing. Surgical treatment is usually reserved for intolerable pain, recurrent ulcerations, infection and joint destruction. Our case series highlights the importance of recognising ulcerated gout in patients presenting with a hot swollen foot mimicking osteomyelitis. A high index of suspicion led to revision of diagnosis and subsequent appropriate management with rapid and satisfactory resolution of ulcerated gout.
\end{abstract}

Key words: diabetes, gout, foot, osteomyelitis, antibiotics

\section{Introduction}

Gout, once described as "a disease afflicting nobility and kings", has made a significant resurgence in recent years affecting people with type 2 diabetes, hyperuricaemia, hypertension and chronic kidney disease. ${ }^{1-4}$ This excruciatingly painful inflammatory arthritis leads to articular/periarticular urate crystals called "tophi" which are inspissated or semiliquid chalky deposits capable of inflicting joint damage. ${ }^{5}$ Gout usually affects the first MTP joint of the foot with other regional monoarticular involvement being less common. ${ }^{6}$ The diagnosis of gout is based on clinical criteria and the identification of negatively birefringent crystals on polarised microscopy. ${ }^{7,8}$ Clinical factors associated

Department of Diabetes \& Endocrinology, University Hospitals of Leicester NHS Trust, Leicester, UK

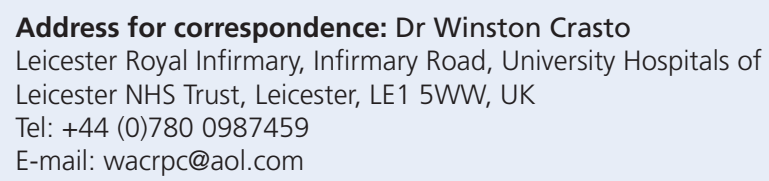

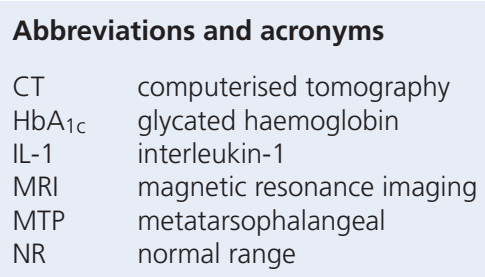

with an increased risk of gout in people with type 2 diabetes are increased age, male gender, smoking, being overweight and impaired kidney function. Conversely, clinical factors associated with a decreased risk of gout in people with type 2 diabetes are significantly high $\mathrm{HbA}_{1 \mathrm{c}}$ levels which may be due to the uricosuric effect of glycosuria as a result of poorly controlled diabetes. ${ }^{9}$

In clinical practice, patients with type 2 diabetes presenting with a hot red swollen foot in the presence of a non healing ulcer are usually presumed to have underlying osteomyelitis and are treated with a prolonged course of antibiotics. Gout is an important differential diagnosis when the initial presentation is a swollen, hot, red and painful foot. However, ulcerated tophaceous gout can masquerade as a non healing diabetic foot ulcer and presents a diagnostic challenge.

In this case series we present our clinical experience and management of three patients presenting with a hot swollen foot with associated non healing ulcers. The patients presented between January 2011 and September 2013 at the multidisciplinary diabetes foot clinic at our tertiary referral centre. Although these patients were initially managed and treated for underlying osteomyelitis, a high index of suspicion led to revision of diagnosis and subsequent management with rapid and satisfactory resolution of ulcerated gout.

\section{Case 1}

A 71 year old Asian man with type 2 diabetes and hypertension (treated with lisinopril) was referred by his general practitioner with a 4 month long history of swelling and minute superficial ulceration on the medial aspect of the right first MTP joint (Figure 1). He had received two courses of empirical antibiotic therapy prior to presentation to the foot clinic. X-ray of the right foot showed widespread destruction of the distal shaft of the first metatarsal and first MTP joint (Figure 2). Biochemical profile showed a white blood cell count of $9100 / \mathrm{ml}$, C-reactive protein $163 \mathrm{mg} / \mathrm{dl}$ (NR: 0 to 10 ) and $\mathrm{HbA}_{1 \mathrm{c}}$ of $61 \mathrm{mmol} / \mathrm{l}(7.7 \%)$. Swabs from the ulcer revealed Staphylococcus aureus and $\beta$ haemolytic streptococcus. The initial clinical suspicion was infected superfi- 
Figure 1. Initial appearance of red hot swollen foot with minute ulceration.

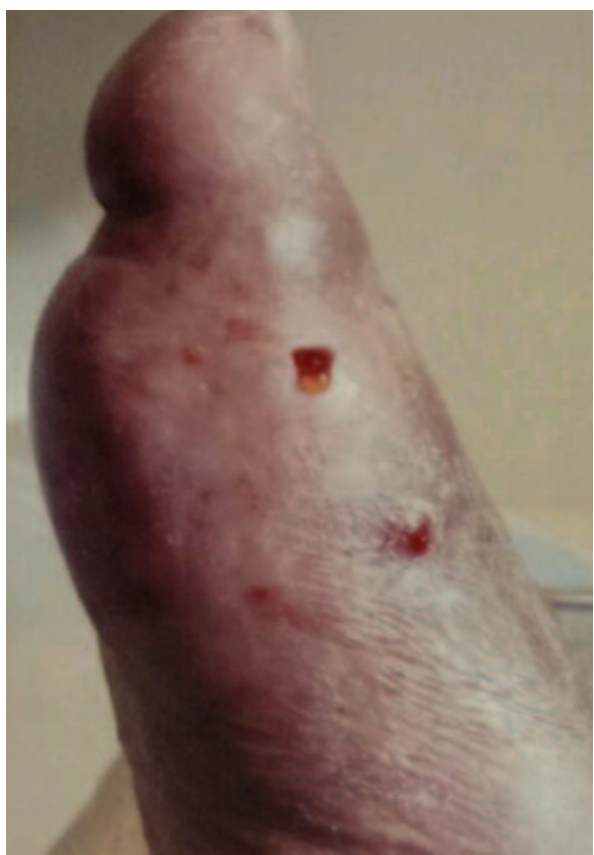

Figure 2. Plain X-ray image of affected foot showed extensive soft tissue swelling around the great toe, associated with destruction of the distal first MTP and first proximal phalanx.

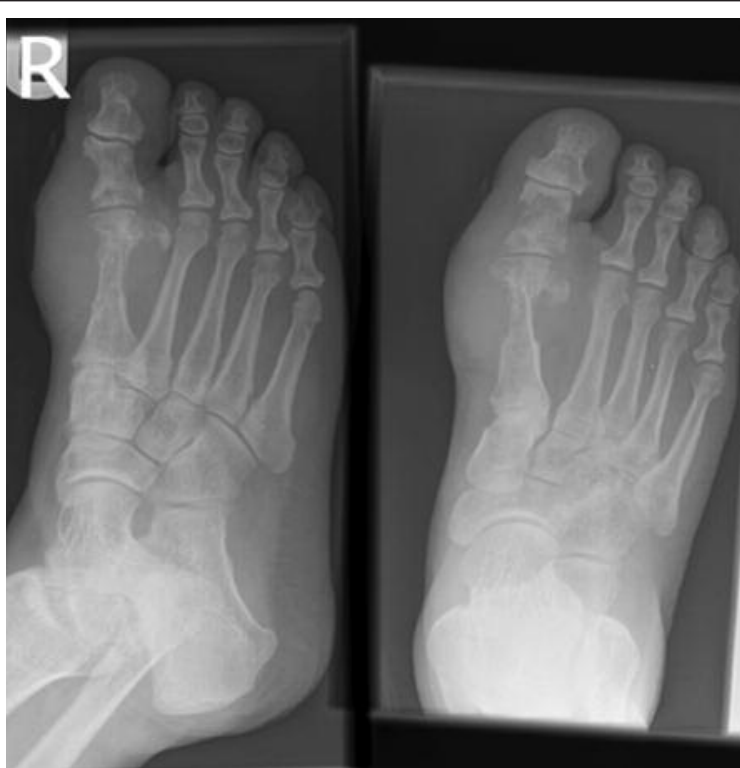

cial diabetic foot ulcers with underlying osteomyelitis and the patient was treated with oral antibiotics for four weeks guided by culture and sensitivity reports. However, there was little suggestion of healing and the foot continued to remain hot and swollen with persistent ulceration. A CT scan of the affected foot
Figure 3. CT scan showing a circumferential bone erosion and soft tissue mass measuring $3.6 \times 4.7 \mathrm{~cm}$ suggestive of tophaceous deposit around the first MTP joint.

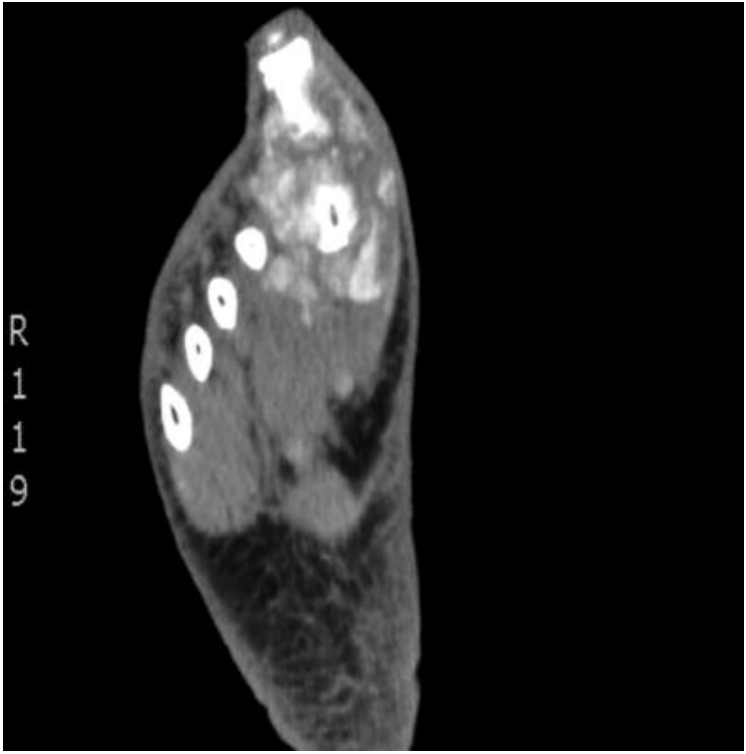

Figure 4. Excision and complete removal of gouty tophaceous mass.

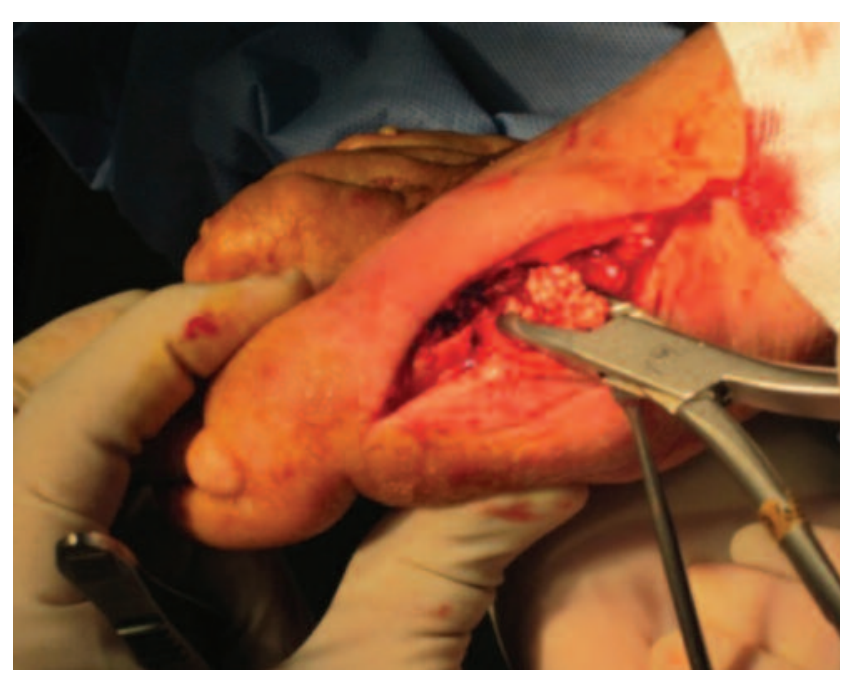

showed circumferential bone erosion and soft tissue mass suggestive of tophaceous deposit around the first MTP joint (Figure

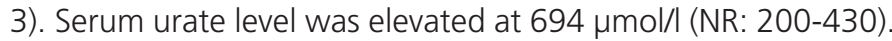
Joint fluid aspiration revealed negatively birefringent crystals.

He was treated for acute gout with colchicine. Allopurinol was commenced once the acute inflammation had subsided. Four weeks later, despite near resolution of the ulcerated area, the patient presented again with a painful swollen foot and tiny punched out ulcers on the base of the fore and mid-foot, both 
Figure 5. Post-operative wound demonstrates complete wound healing at 2 months.

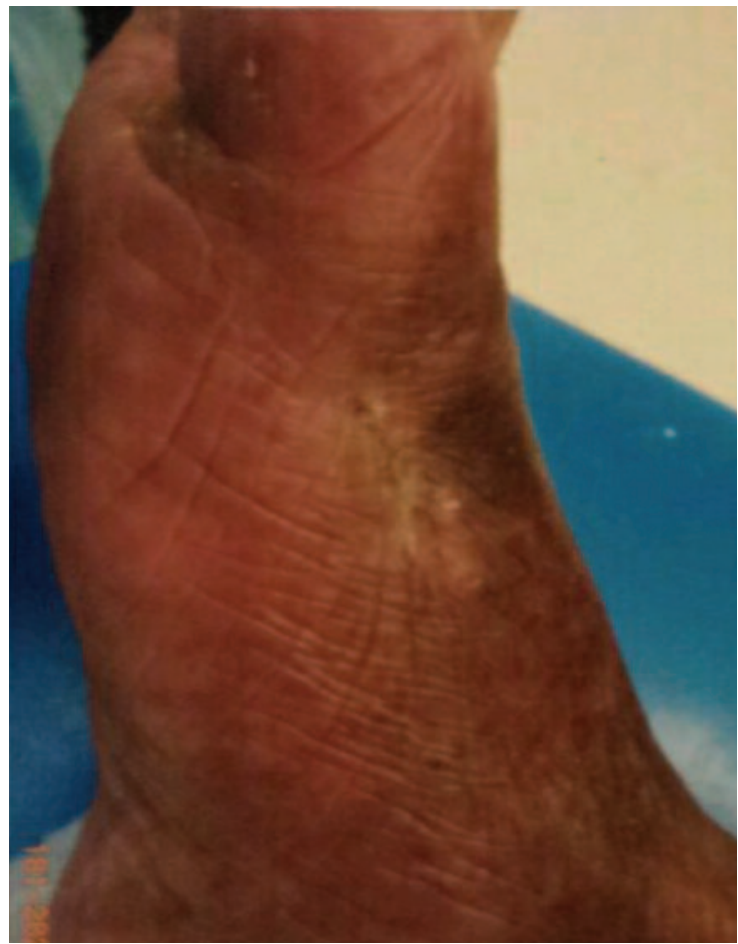

interconnected and probing to bone. Radical surgical debridement with complete removal of the tophaceous mass was carried out and 2 months post operatively, there was complete wound healing (Figures 4,5) and the serum urate level had come down to $481 \mu \mathrm{mol} / \mathrm{l}$.

\section{Case 2}

A 72 year old man was referred to the diabetes multidisciplinary foot clinic with a 3 week history of a swollen left hallux. He was treated for foot cellulitis with flucloxacillin. His past medical history included type 2 diabetes complicated by diabetic peripheral neuropathy, peripheral vascular disease and a previous episode of gout about 8 years ago. He had an endarterectomy of the left limb 2 months previously which was complicated post operatively by deep vein thrombosis in the same limb. His medication regimen included metformin, allopurinol, aspirin and a beta blocker with the recent addition of warfarin. On examination, he had a swollen erythematous left first MTP joint (Figure 6). In view of his previous clinical history, clinical appearance and site of the swelling, gout was suspected. Incision and drainage was carried out by our podiatric surgeon and the presence of gouty tophi was confirmed. However despite repeat aspiration of the joint, colchicine and increased analgesia, our patient experienced intolerable pain with recurrence of foot swelling. A thorough surgical debridement after stopping warfarin therapy was carried out with removal of gouty tophi. Further review 2 weeks later showed complete resolution of symptoms.
Figure 6. Swollen, hot left first MTP joint.

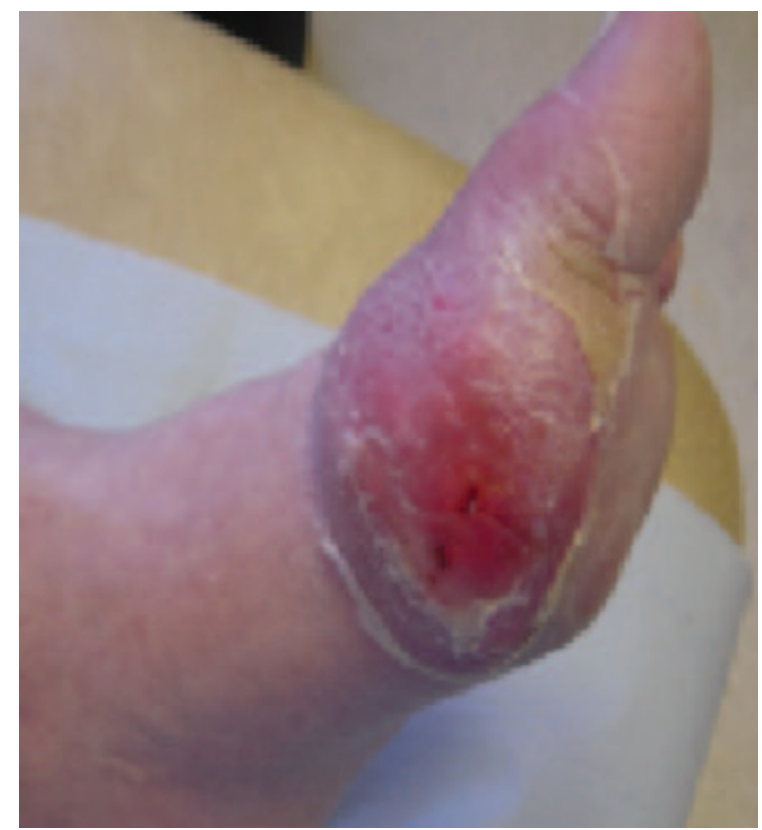

Figure 7. Drainage of chalky inspissated secretions.

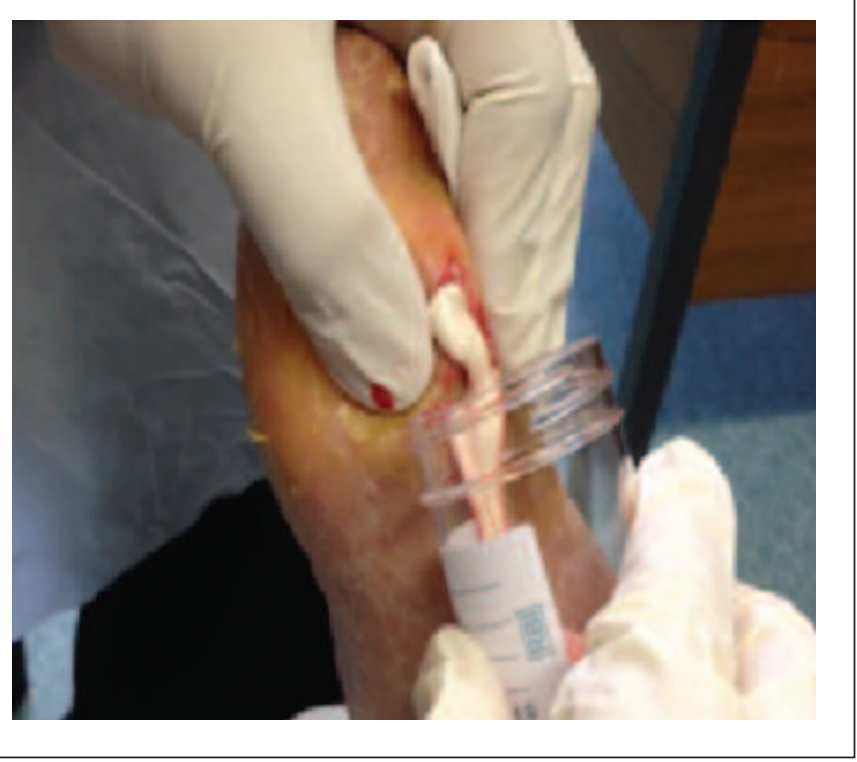

\section{Case 3}

An 83 year old man with type 2 diabetes presented to the multidisciplinary foot clinic with bilateral foot ulcers. He was recovering from a ray amputation of the left first toe due to osteomyelitis of the first metatarsal head but had developed new minute ulcers on the medial aspect of the right hallux. At presentation the patient had received antibiotic therapy for nearly four months in the community. There was suspicion of underlying osteomyelitis in the right foot in the presence of non healing ulcers. The most prominent clinical finding was a red, hot and 
Figure 8. X-ray of the right foot showing soft tissue swelling, marked joint destruction and juxta articular erosion.

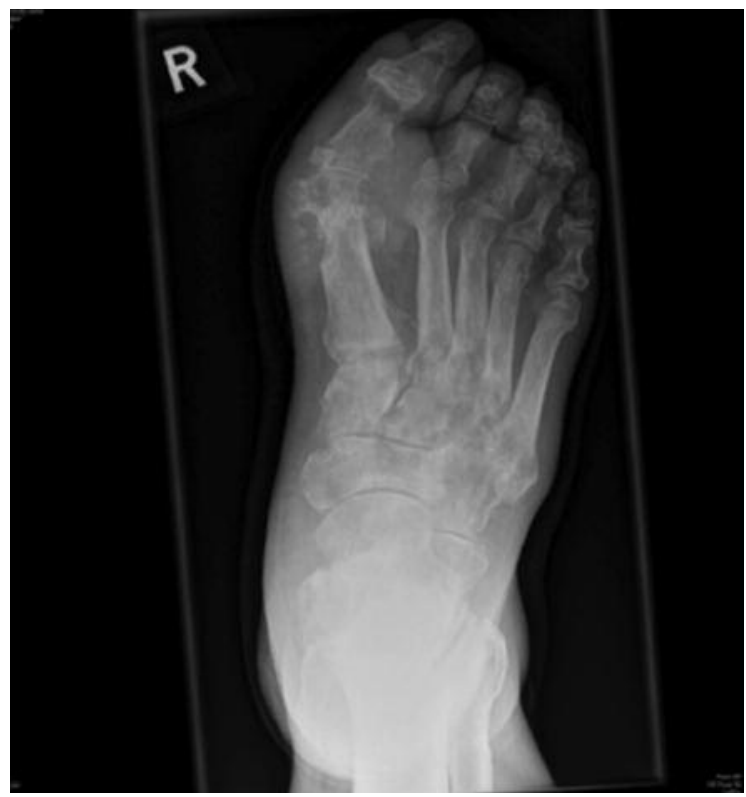

swollen right first MTP joint. A high index of suspicion led to immediate incision and drainage. A copious quantity of chalky semi-liquid material was drained (Figure 7). The diagnosis of gout was confirmed by the presence of negatively birefringent crystals under polarised light microscopy. A thorough surgical debridement was carried out with complete removal of underlying gouty tophi which had invaded the underlying bone and joint space resulting in significant bone and joint destruction (Figure 8).

\section{Discussion}

The patients described in this case series presented to our multidisciplinary diabetes foot clinic principally with a red hot, swollen foot with ulceration and were initially managed for presumed foot infection and osteomyelitis. In the event of non healing or poor wound healing despite optimised management after four to six weeks, other differentials must be considered. These may include the persistence of bone infection from inability of antibiotic therapy to penetrate and concentrate in the infected bone, poor response or inadequate susceptibility to antibiotic therapy, or poor compliance with treatment which must also be considered. Our case series highlights the importance of considering ulcerated gout which, although uncommon, is an important differential for a non healing diabetic foot ulcer.

The association of hyperuricaemia, gout and diabetes is well known. In particular the presence of gout and type 2 diabetes may suggest a high risk cardiovascular state and requires strict control of cardiovascular risk factors. ${ }^{10}$ In patients with type 2 diabetes, the modulation of serum uric acid concentration influenced by the presence of insulin resistance, chronic renal failure, obesity and/or diuretic therapy including loop diuretics, furosemide and thiazide diuretics, which are often prescribed in this patient group, are responsible for hyperuricaemia and can lead to an increased incidence of gouty arthritis. ${ }^{11,12}$

A definitive diagnosis of gout is made by observation of negatively birefringent monosodium urate crystals in synovial fluid aspirate or tophi which has a sensitivity of $85 \%$ and specificity of $100 \% .^{5,8}$ Sodium urate and uric acid possess bacteriostatic properties and concomitant sepsis is usually mild and well localized. However, ulcerated gout can be complicated by the presence of concomitant Staphylococcus aureus infection which requires antibiotic therapy. ${ }^{13}$ Plain radiography, CT, MRI, ultrasound and bone scintigraphy are useful imaging modalities to assess complications and monitor disease progression and may indicate chronicity of the disease process, but they are not useful for a definitive diagnosis of gout. ${ }^{14}$ Plain X-ray images are not sufficiently sensitive and specific since typical "punched out," periarticular erosions with overhanging appearance are seldom seen at least until 6 to 8 years after an acute attack of gout. ${ }^{15}$ MRI appearances of tophaceous gout can be variable on T2weighted images, appearing as heterogeneously low to intermediate signal intensity depending on the calcium burden within a tophus. The presence of a mass deposit with typical erosive changes and involvement of adjacent bone or joints however is highly suggestive. ${ }^{16}$

Surgical treatment in ulcerated gout is usually reserved for sepsis or infection of ulcerated tophi, large tophaceous mass, draining sinus, to relieve pain and for recurrent flare-ups resulting in joint deformity and joint destruction. ${ }^{17}$ Forceful curettage of gouty tophi may compromise local circulation and simple enucleation of the tophi may lead to complications such as skin necrosis, tendon and joint exposures. ${ }^{18}$ Surgical approaches for removal of gouty deposits include soft-tissue shaver-assisted intra-lesion excavating technique, ${ }^{18,19}$ debridement of tophi by applying highly pressurized saline stream and complex surgical approaches involving large skin incisions and excision of the tophi. ${ }^{20}$ Resection of joints, arthroplasty, and arthrodesis of painful joints may lessen pain and improve function of the affected limb. Medical management including dietary and lifestyle modifications, anti-inflammatory drugs, colchicine, and uricosuric agents such as probenecid or those which inhibit uric acid production such as allopurinol and febuxostat can help to prevent complications and acute attacks following surgery. 5,21 Other treatment modalities for acute attacks refractory to classical treatment include the use of oral or topical steroids, corticotropin (ACTH), IL-1 antagonists and monoclonal antibody against IL-1 $\beta .5$

\section{Conclusion}

Although uncommon, ulcerated gout should be considered in patients presenting with a non healing diabetic foot ulcer, particularly if the first hallux is involved with evidence of bone involvement seen on X-ray or other imaging studies. The diagnosis may be suggestive of gout with a previous history of hyperuricaemia. Early recognition of ulcerated gout which can masquerade as diabetic foot infection, tissue sampling, imaging modalities and prompt institution of aggressive medical treatment can avoid further invasion and destruction of adjacent 


\section{Key message}

- Osteomyelitis is often considered in a patient with diabetes presenting with a red hot swollen foot and a non healing ulcer. An important differential diagnosis is ulcerated gout, a relatively uncommon condition which can masquerade as a non healing diabetic foot ulcer and presents a diagnostic challenge

musculoskeletal structures and aid prompt healing. Surgical treatment in ulcerated gout is usually reserved for intolerable pain, recurrent ulcerations, infection and joint destruction. Optimal medical management helps to prevent recurrence and avoid complications.

\section{Conflict of interest None Funding sources None}

Acknowledgements We thank Dr Deborah Modha, consultant microbiologist, Rachel Berrington, diabetes specialist nurse and foot care lead and Dr Mary Quinn, diabetes associate specialist, for their help in the management of these patients.

\section{References}

1. Abbott CA, Carrington AL, Ashe H, et al, and North-West Diabetes Foot Care Study. The North-West Diabetes Foot Care Study: incidence of, and risk factors for, new diabetic foot ulceration in a community-based patient cohort. Diabet Med 2002;19:377-84. http://dx.doi.org/10.1046/j.1464-5491.2002.00698.x

2. Lai HM, Chen CJ, Su BY-J, Chen YC, et al. Gout and type 2 diabetes have a mutual inter-dependent effect on genetic risk factors and higher incidences. Rheumatology 2012;51:715-20. http://dx.doi.org/10.1093/rheumatology/ker373

3. Rothenbacher D, Primatesta P, Ferreira A et al. Frequency and risk factors of gout flares in a large population-based cohort of incident gout. Rheumatology 2011;50:973-81. http://dx.doi.org/10.1093/rheumatology/keq363

4. Suppiah R, Dissanayake A, Dalbeth N. High prevalence of gout in patients with type 2 diabetes: male sex, renal impairment, and diuretic use are major risk factors. N Z Med J 2008;121:43-50.

5. Neogi T. Clinical practice. Gout. N Engl J Med 2011;364:443-52. http://dx.doi.org/10.1056/NEJMcp1001124

6. Iwamoto T, Toki $\mathrm{H}$, Ikari $\mathrm{K}$ et al. Multiple extensor tendon ruptures caused by tophaceous gout. Mod Rheumatology 2010;20:210-12. http://dx.doi.org/10.3109/s10165-009-0258-x

7. Chen LX, Schumacher HR. Current trends in crystal identification. Curr Opin Rheum 2013;18:171-3. http://dx.doi.org/10.1097/01.bor. 0000209430.59226.0f

8. Wallace SL, Robinson H, Masi AT, et al. Preliminary criteria for the classification of the acute arthritis of primary gout. Arthrit Rheum 1997;20:895-900. http://dx.doi.org/10.1002/art.1780200320

9. Rodriguez G, Soriano L, Choi H. Impact of diabetes against the future risk of developing gout. Ann Rheum Dis 2010;69:2090-4. http://dx.doi.org/10.1136/ard.2010.130013

10. Choi HK, De Vera MA, Kishnan E. Gout and risk of type 2 diabetes among men with a high cardiovascular risk profile. Rheumatology 2008;47:1567-70. http://dx.doi.org/10.1093/rheumatology/ken305

11. Wordsworth BP, and Mowat AG. Rapid development of gouty tophi after diuretic therapy. J Rheumatol 1985;12:376-77.

12. Facchini F, Chen Y, Hollenbeck C, Reaven G. Relationship between resistance to insulin-mediated glucose uptake, urinary uric acid clearance, and plasma uric acid concentration. JAMA 1991;266:3008-11. http://dx.doi.org/10.1001/jama.1991.03470210076036

13. Yu KH, Luo SF, Liou LB, et al. Concomitant septic and gouty arthritis-an analysis of 30 cases. Rheumatology 2003;42:1062-66. http://dx.doi.org/ 10.1093/rheumatology/keg297

14. Dalbeth N, McQueen FM. Use of imaging to evaluate gout and other crystal deposition disorders. Curr Opin Rheumatol 2009;21:124-31. http://dx.doi.org/10.1097/BOR.0b013e3283257b6c

15. Brower AC, Flemming DJ. Gout. In: Arthritis In Black and White. 2nd ed. Philadelphia: WB Saunders; 1997:325-341.

16. Yu J, Chung C, Recht M, et al. MR imaging of tophaceous gout. Am J Roentgenology 1997;168:523-7. http://dx.doi.org/10.2214/ajr.168.2.9016240

17. Kumar S, Gow P. A survey of indications, results and complications of surgery for tophaceous gout. NZ Med J 2002;115:U109.

18. Lee SS, Chen MC, Chou YH, et al. Timing of intra-lesion shaving for surgical treatment of chronic tophus. J Plast Reconstr Aesthet Surg 2013; 66:1131-37. http://dx.doi.org/10.1016/j.bjps.2013.03.041

19. Lee SS, Sun IF, Lu YM, Chang KP, et al. Surgical treatment of the chronic tophaceous deformity in upper extremities - the shaving technique. J Plast Reconstr Aesthet Surg 2009;62:669-74. http://dx.doi.org/10.1016/j.bjps.2007.12.021

20. Lee JH, Park JY, Seo JW, et al. Surgical treatment of subcutaneous tophaceous gout. J Plast Reconstr Aesthet Surg 2010;63:1933-35. http://dx.doi.org/10.1016/j.bjps.2010.03.019

21. Larmon W, Kurtz J. The Surgical Management of Chronic Tophaceous Gout. J Bone Joint Surg 1958;40:743-72. 